\title{
Gaia TGAS search for Large Magellanic Cloud runaway supergiant stars
}

\section{Candidate hypervelocity star discovery and the nature of $\mathbf{R} 71$}

\author{
Daniel J. Lennon ${ }^{1}$, Roeland P. van der Marel$^{2}$, Mercedes Ramos Lerate ${ }^{3}$, \\ William O'Mullane ${ }^{1}$, and Johannes Sahlmann ${ }^{1,2}$

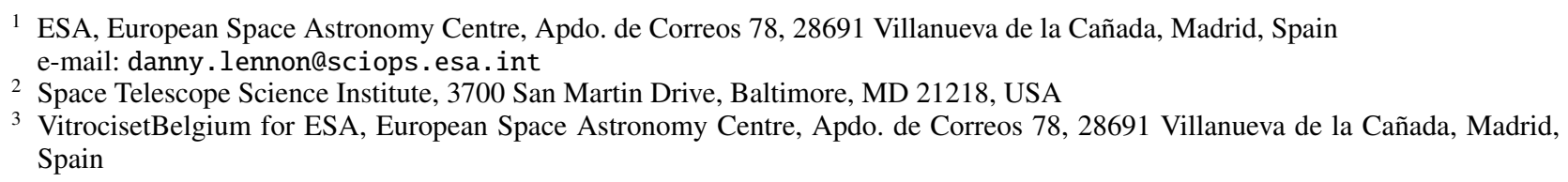

Received 16 November 2016 / Accepted 18 March 2017

\begin{abstract}
Aims. Our research aims to search for runaway stars in the Large Magellanic Cloud (LMC) among the bright HIPPARCos supergiant stars included in the Gaia DR1 Tycho-Gaia astrometric solution (TGAS) catalogue.

Methods. We compute the space velocities of the visually brightest stars in the Large Magellanic Cloud that are included in the TGAS proper motion catalogue. This sample of 31 stars contains a luminous blue variable (LBV), emission line stars, blue and yellow supergiants, and an $\mathrm{SgB}[\mathrm{e}]$ star. We combine these results with published radial velocities to derive their space velocities, and by comparing with predictions from stellar dynamical models we obtain each star's (peculiar) velocity relative to its local stellar environment.

Results. Two of the 31 stars have unusually high proper motions. Of the remaining 29 stars we find that most objects in this sample have velocities that are inconsistent with a runaway nature, being in very good agreement with model predictions of a circularly rotating disk model. Indeed the excellent fit to the model implies that the TGAS uncertainty estimates are likely overestimated. The fastest outliers in this subsample contain the LBV R 71 and a few other well known emission line objects though in no case do we derive velocities consistent with fast $\left(\sim 100 \mathrm{~km} \mathrm{~s}^{-1}\right)$ runaways. On the contrary our results imply that $\mathrm{R} 71$ in particular has a moderate deviation from the local stellar velocity field $\left(40 \mathrm{~km} \mathrm{~s}^{-1}\right)$ lending support to the proposition that this object cannot have evolved as a normal single star since it lies too far from massive star forming complexes to have arrived at its current position during its lifetime. Our findings therefore strengthen the case for this LBV being the result of binary evolution. Of the two stars with unusually high proper motions we find that one, the isolated B1.5 Ia ${ }^{+}$supergiant Sk-67 2 (HIP 22237), is a candidate hypervelocity star, the TGAS proper motion implying a very large peculiar transverse velocity $\left(\sim 360 \mathrm{~km} \mathrm{~s}^{-1}\right)$ directed radially away from the LMC centre. If confirmed, for example by Gaia Data Release 2, it would imply that this massive supergiant, on the periphery of the LMC, is leaving the galaxy where it will explode as a supernova.
\end{abstract}

Key words. Magellanic Clouds - stars: massive - supergiants - stars: kinematics and dynamics - proper motions

\section{Introduction}

A new model of the stellar dynamics of the Large Magellanic Cloud (LMC) was presented by van der Marel \& Sahlmann (2016, from now on vdMS) based on the proper motions of 29 HIPPARCos stars that are LMC members and that have suitably precise proper motions published in the TGAS catalogue of the first Gaia Data Release DR1 (Gaia Collaboration 2016a,b; Lindegren et al. 2016). Besides their use as test particles of the LMC velocity field, these stars are of great intrinsic interest due to their representing a sample of the visually brightest stars in a nearby star forming galaxy. This selection of blue and yellow supergiant stars (see Table 1 and Fig. 1) includes a number of well known peculiar and interesting objects such as the luminous blue variable (LBV) R 71, an SgB[e] star (Sk-69 46), a candidate LBV (Sk-69 75; Prinja \& Schild 1991), and some peculiar emission line objects such as HD 37836 (Shore \& Sanduleak 1984).
The dynamical properties of these massive stars are of interest. Those field stars that are relatively isolated, being far from young clusters, are of particular significance since there are competing hypotheses to explain their solitude. Possible explanations include in situ formation of single massive stars (Parker \& Goodwin 2007), or ejection of runaway stars (with peculiar velocities of the order of $100 \mathrm{~km} \mathrm{~s}^{-1}$ ) via disruption of a close binary by a supernova (SN) explosion (Blaauw 1961) or dynamical interaction in a massive cluster (Poveda et al. 1967). Indeed de Mink et al. $(2012,2014)$ point out that binary evolution channels enable a continuum of runaway velocities, even including slow runaways, the so-called walkaways. At the other extreme, even higher peculiar velocities (hundreds of $\mathrm{km} \mathrm{s}^{-1}$ ) are thought to be generated through interaction with the supermassive black hole (SMBH) at the centre of our Milky Way (Brown 2015) producing hypervelocity stars, although alternative mechanisms are suggested with the discovery of hypervelocity B-stars that do not fit this paradigm (Przybilla et al. 2008). Clearly the 


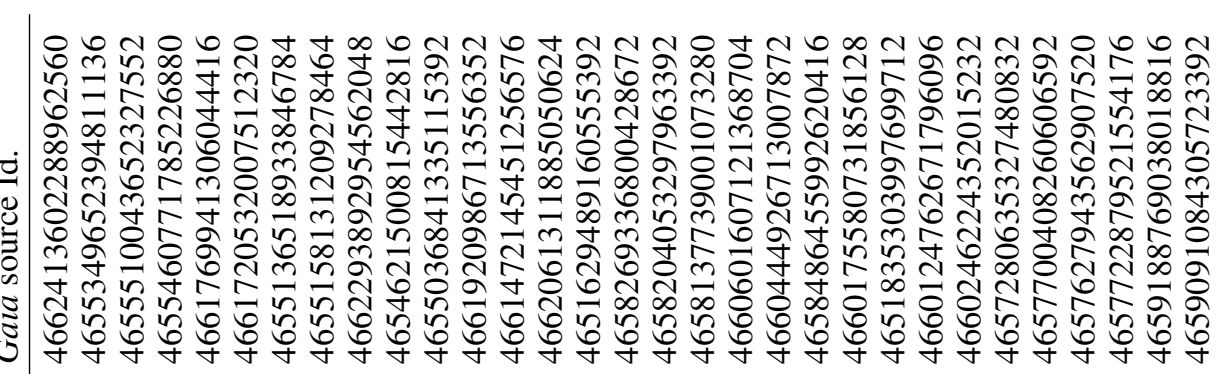

尝

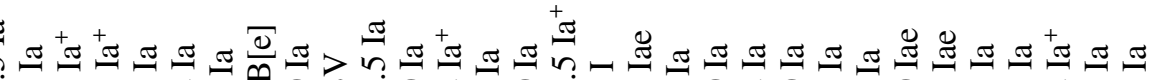

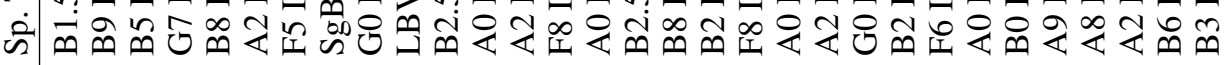

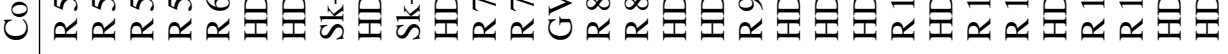
ஜ ॠँ

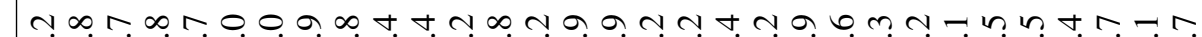

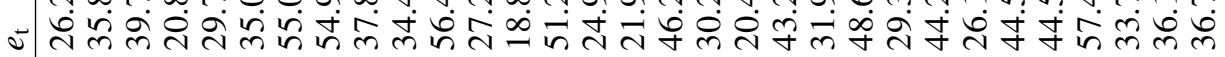
命 z z n そn

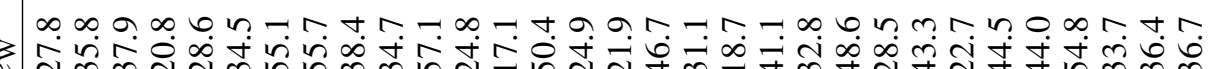

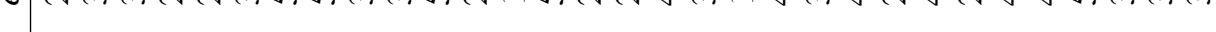

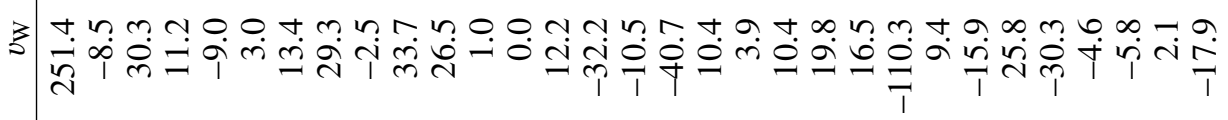

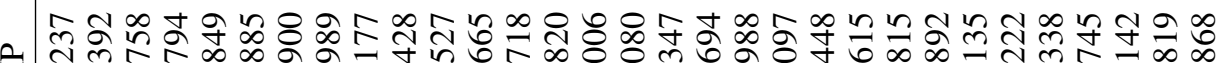
寻 ปิ ปก⿻

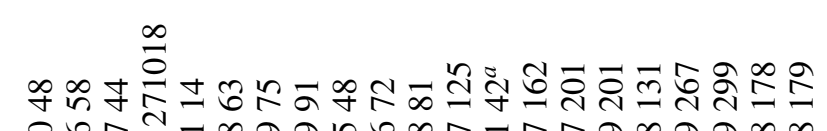

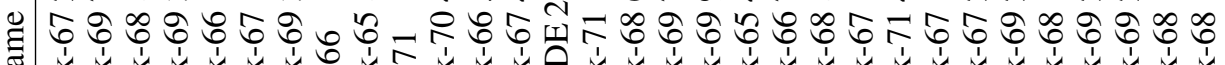

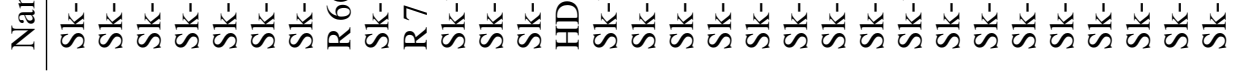

형

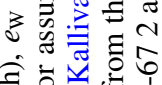

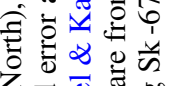

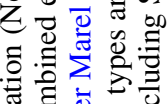

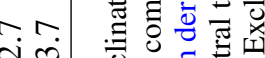

ป்่

$\stackrel{0}{0}$

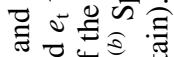




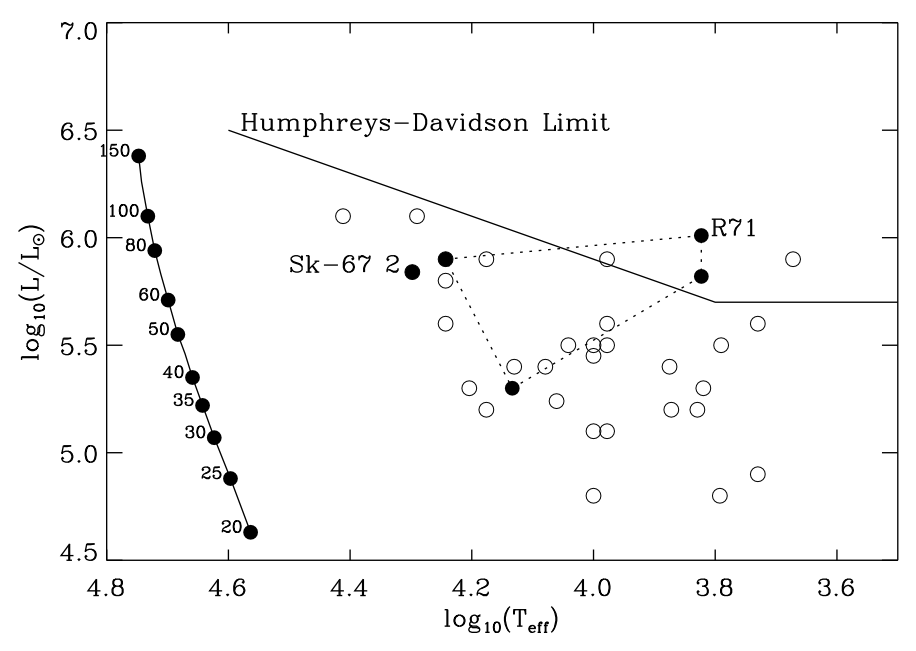

Fig. 1. Open symbols represent positions of the stars in our LMC sample in the HR diagram. Effective temperatures and luminosities are taken from the literature where available, or estimated using spectral types and published photometry. As many of these stars are photometric and spectroscopic variables their positions in the HR diagram are illustrative. As an extreme example the positions of the LBV R 71 are indicated (data from Mehner et al. 2013) joined by dotted lines. For convenience we indicate the position of the candidate hypervelocity star Sk-672. We also indicate the position of the zero age main sequence (left, labelled with initial masses, tracks from Brott et al. 2011; and Köhler et al. 2015) and the approximate location of the Humphreys-Davidson limit.

dynamical signature of these processes is a crucial discriminant in disentangling these scenarios.

Within the Magellanic Clouds the investigation of runaway stars (in general) has been limited mainly to circumstantial evidence such as apparent isolation, peculiar radial velocity, or the presence of an infrared excess that might be attributed to the presence of a bow shock (Evans et al. 2010; Gvaramadze et al. 2010). As a specific example we point to the current debate as to whether some or all LBVs are runaway stars. Smith \& Tombleson (2015) suggest that LBVs are the "kicked mass gainers" in binary evolution arguing that their relative isolation is inconsistent with single star evolution. Although this hypothesis is heavily debated (see Humphreys et al. 2016; Smith 2016; Davidson et al. 2016) it is clear that the evidence is circumstantial since proper motions for stars in the LMC cannot yet discriminate between various propositions (but see Platais et al. 2015 , for a first attempt to measure precise relative proper motions of massive stars around 30 Doradus). The Gaia mission is set to change this picture and fortunately, as noted above, our sample contains the most isolated LBV in the LMC, R71, as well as a number of emission line objects potentially related to LBVs.

In this paper we use the proper motions from the Gaia TGAS catalogue, along with published radial velocities to estimate stellar velocities relative to their local mean stellar velocity field in order to search for runaway stars. In Sect. 2 we describe our methods and discuss the results for the sample as whole, while in Sect. 3 we focus on a few individual objects that are of special interest.

\section{Stellar relative velocities}

Cross-matching the TGAS catalogue with the master catalogue of LMC massive stars from Bonanos et al. (2009), supplemented by the full Sanduleak catalogue (Sanduleak 1970) of bright LMC members, reveals 311 sources in common. Examination of the uncertainties in the measured proper motions reveals two clear groupings; 31 sources, all HIPPARCOS sources, with proper motion uncertainties less than approximately $0.25 \mathrm{mas} / \mathrm{yr}$, and all others, the Tycho sources, with uncertainties in excess of approximately $0.6 \mathrm{mas} / \mathrm{yr}$. In vdMS the TGAS proper motions of 29 of these 31 HIPPARCos sources were used to improve the three-dimensional model of the rotation field of that galaxy (see also Kroupa \& Bastian 1997). This work built upon the previous work of van der Marel \& Kallivayalil (2014) that modelled this field as a rotating flattened disk, constrained by 6790 lineof-sight (LOS) stellar velocities and the average proper motions of 22 fields in the LMC as derived from Hubble Space Telescope (HST) observations. Implicit in the use of these 29 stars to constrain this model is the expectation that their velocities are a reasonable reflection of the mean stellar motions at their respective positions. It follows that, say, any runaway star present in this sample, with a peculiar relative velocity in excess of around $50 \mathrm{~km} \mathrm{~s}^{-1}$ (about $0.2 \mathrm{mas} / \mathrm{yr}$ in the LMC), would have larger residuals in the model fit than stars that are not runaway stars. In this context we note that the formal errors in the proper motions of these stars presented in TGAS have a mean value of $\sim 0.16$ mas/yr in both right ascension and declination. While this is $\sim 37 \mathrm{~km} \mathrm{~s}^{-1}$ in velocity terms (see Table 1), as we discuss below we have reason to believe that the actual errors are substantially smaller than this. Regardless of this latter point, the precision of the TGAS proper motions is even now sufficient to search for (fast) runaway stars among our sample.

We therefore compute the residuals between each star's proper motion and the vdMS model prediction, as a measure of its velocity relative to the mean motion of stars in the LMC. We also compute the peculiar LOS velocity by comparing their radial velocities with the model estimates as constrained by van der Marel \& Kallivayalil (2014, see their Fig. 4). These results are listed in Table 1 where we have converted proper motion to velocity assuming a distance to the LMC of $50.1 \pm 2.5 \mathrm{kpc}$, corresponding to a distance modulus of $\mathrm{m}-M=18.50 \pm 0.1$ (Freedman et al. 2001). We note that the distance uncertainty also implies a $\sim 5 \%$ systematic uncertainty in predicted and derived velocities. In Table 1 we also include two stars, HIP 22237 (Sk-67 2) and HIP 25815 (Sk-71 42), that were excluded by vdMS on the basis that their proper motions are unusual and their excess_astrometric_noise parameter is large compared to other stars in the sample. We have included these two objects in our analysis and will return to them in the next section. For now we point out that their resultant peculiar velocities are very large, more akin to hypervelocity stars, and we exclude them from the following discussion of the statistical properties of the sample of 29 stars. However, we will return to these two objects in the next section, while the measured proper motions of all 31 stars are illustrated in Fig. 2, that also serves as context for insight into each star's local environment within the LMC.

In Fig. 3 we illustrate our results as velocity dispersion histograms in each of the three directions of motion. One of the striking aspects of the overall results is that the goodness of fit to the model is excellent; there are no outliers beyond $50 \mathrm{~km} \mathrm{~s}^{-1}$ in right ascension or declination (see also Table 1). In fact the standard deviations of the residuals in right ascension and declination are each approximately $19 \mathrm{~km} \mathrm{~s}^{-1}$, substantially less than the value that might be expected given that the formal proper motion errors predict a mean of $37 \mathrm{~km} \mathrm{~s}^{-1}$ in velocity as mentioned above (well outside the 5\% uncertainty in the distance to the LMC). Figure 4 shows the residual vectors in a polar plot indicating that there is no preferred direction for the peculiar velocities. We therefore interpret the proper motions of the present 


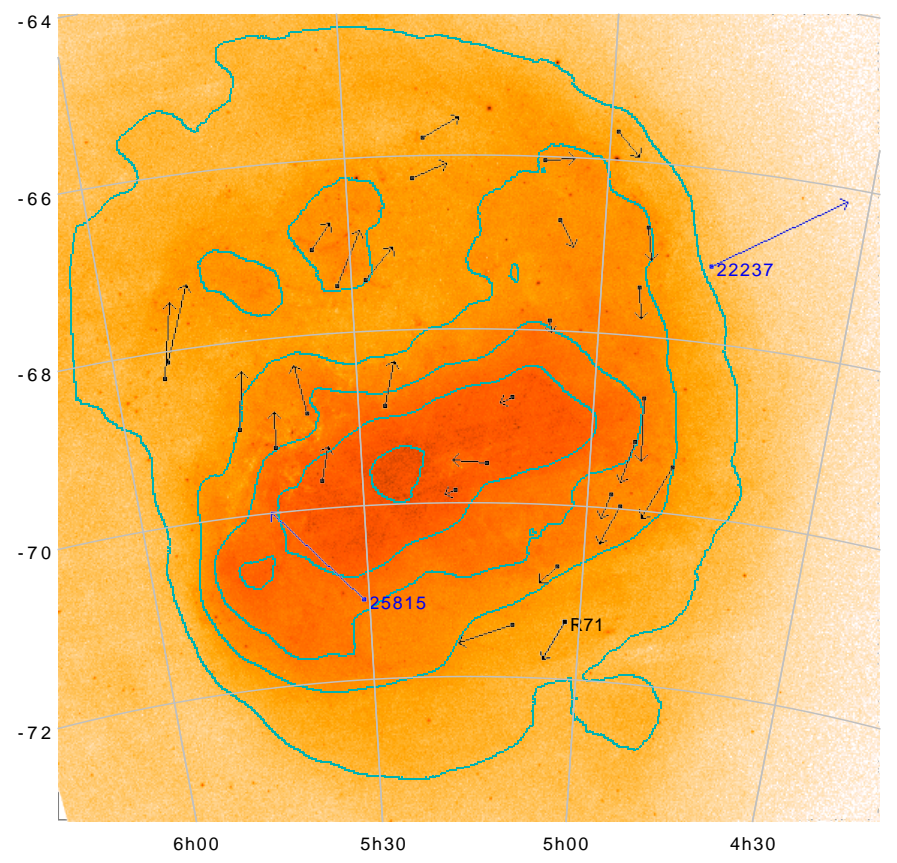

Fig. 2. Black arrows indicate magnitudes and directions of proper motions of the 29 stars from vdMS (the LBV R 71 is labelled), while blue arrows denote positions and proper motions of Sk-67 2 (HIP 22237) and Sk-71 42 (HIP 25815) as indicated by the labels. The background image illustrates the stellar density map and contours for the LMC derived from the Gaia catalogue.

sample of stars as implying that they do not harbour fast runaways (with peculiar velocities in excess of $50 \mathrm{~km} \mathrm{~s}^{-1}$ ).

The LOS velocity information is more difficult to interpret since measured radial velocities are subject to a number of effects that introduce off-sets from the true LOS velocity. All of these stars are very luminous (in the approximate range $10^{5}-$ $10^{6}$ solar luminosities) and therefore many have strong winds that will affect the centroids of strong absorption lines and emission lines. The impact on the former is small, typically introducing a small blue-shift of $\sim 10 \mathrm{~km} \mathrm{~s}^{-1}$ (Arp 1992), while pure emission lines may be even more blue-shifted since they form in the outflowing wind (see the discussion of the forbidden [Fe II] lines of R 71 in the next section, and the discussion of the radial velocity of the suspected runaway or walkaway VFTS 682 near 30 Doradus by Bestenlehner et al. 2011). The details of course will depend crucially on the parameters of the stellar wind (stellar radius, mass-loss rate, terminal velocity, and velocity law). Multiplicity can also play a role (it is known that Sk-68 83 is an eclipsing binary with a semi-amplitude of $\sim 35 \mathrm{~km} \mathrm{~s}^{-1}$ ) and will in general lead to an overestimate of the velocity dispersion if ignored (Hénault-Brunet et al. 2012). However, the multiplicity characteristics of these types of stars are poorly understood at present, and many stars of our sample have only single epoch measurements. Given these caveats, and also noting the heterogeneous nature of the sources for the radial velocities, it is perhaps not too surprising to note that the velocity dispersion derived here of $\sim 20 \mathrm{~km} \mathrm{~s}^{-1}$ is larger than the $11.6 \mathrm{~km} \mathrm{~s}^{-1}$ derived by van der Marel \& Kallivayalil (2014) for young red supergiants using the same model.

Summarising the results of this section, with the exception of the two anomalous stars Sk-67 2 and Sk-71 42, the proper motions and LOS velocities of this sample are consistent with the hypothesis that none are fast runaways, though there are some outliers that may be slower runaway stars with peculiar space

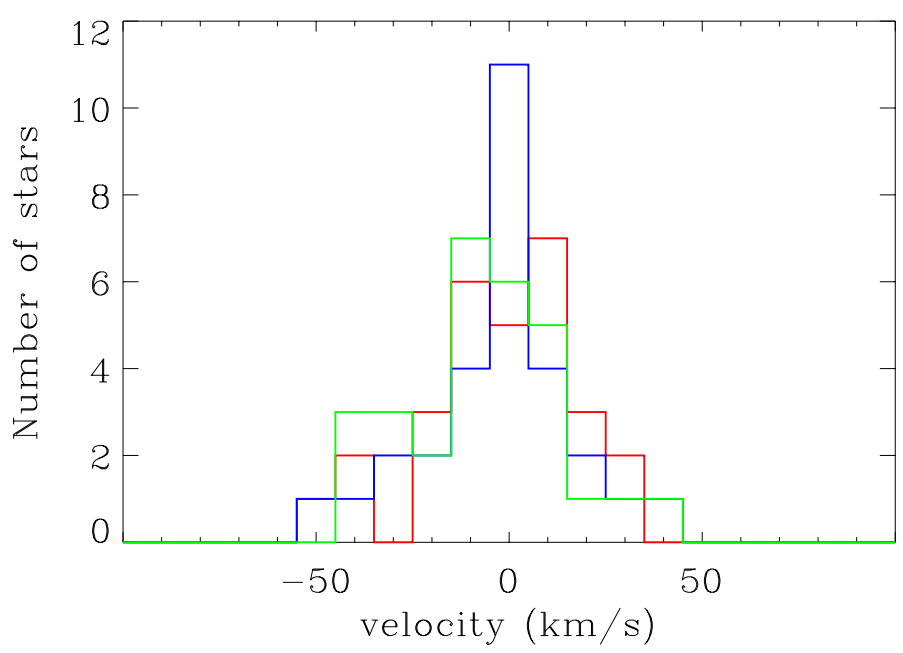

Fig. 3. Illustrated here are the velocity residuals in right ascension $\left(v_{\mathrm{W}}\right.$, red $)$, declination $\left(v_{\mathrm{N}}\right.$, blue $)$, and along the LOS ( $v_{\mathrm{LOS}}$, green). Histograms have a bin size of $10 \mathrm{~km} \mathrm{~s}^{-1}$ and the mean peculiar velocities in $\mathrm{W}, \mathrm{N}$, and LOS directions are $2.8,1.5$, and $-0.9 \mathrm{~km} \mathrm{~s}^{-1}$ respectively, with standard deviations of $18.9,18.8$, and $20.3 \mathrm{~km} \mathrm{~s}^{-1}$. Sk-67 2 (HIP 22237) and Sk-71 42 (HIP 25815) are not included in these figures or estimates.
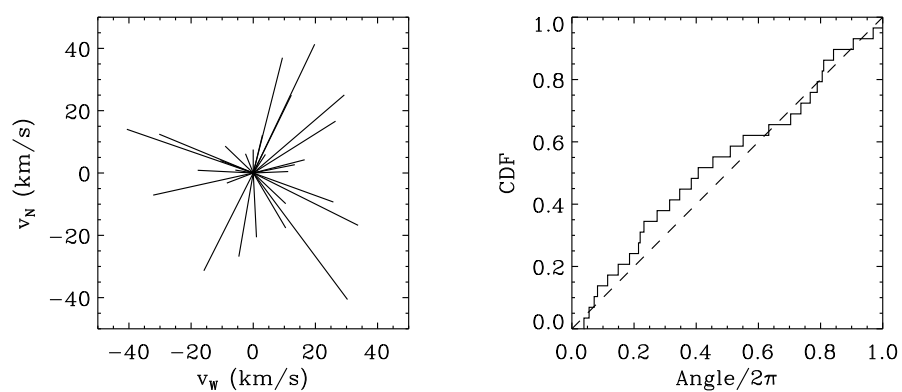

Fig. 4. Left panel: plot of $v_{\mathrm{W}}$ and $v_{\mathrm{N}}$ (from Table 1); right panel: cumulative distribution function (CDF) of the position angles of these vectors as a histogram. The straight dashed line represents the CDF of a random distribution. A formal comparison of the two distributions using the Kuiper test indicates that the observations are consistent with this random distribution at a significance level of $85 \%$.

velocities around $50 \mathrm{~km} \mathrm{~s}^{-1}$. A subset of the five fastest slow runaways includes the isolated B-hypergiant Sk-68 8, the LBV R 71, the candidate LBV Sk-6975, and the SgB[e] star R 66.

\section{Discussion: Individual objects of special interest}

In this section we discuss in more detail some implications of our results for a few objects of special interest.

\section{1. $R 71$ (=Sk-71 3, HDE 269006)}

The well studied LBV R 71 has in recent years been undergoing an outburst, reaching a maximum visual magnitude of $\sim 8.5$ during the year 2012. It is particularly interesting as it is the most isolated LBV in the LMC, and is also one of the least luminous of this class. However, we note the influence of assumptions concerning extinction as pointed out by Mehner et al. (2013), where more details on this object's recent activity may be found.

There has been a recent suggestion by Smith \& Tombleson (2015) that LBVs, such as R 71, are the rejuvenated mass gainer products of massive star binary evolution. Central to this idea is their proposition that LBVs tend to be found in the field and well 
away from massive star clusters, a consequence of a more massive star donating material to the initially lower mass companion, before exploding as a SN and kicking the now more massive star out of its parent cluster. This runaway star subsequently evolves into its LBV phase though now as a relatively isolated star, before finally exploding as a SN far from its parent cluster. This is disputed by Humphreys et al. (2016) who argue that LBVs are predominantly the products of single star evolution arguing that the locations of massive LBVs are well correlated with O-type stars, while the locations of less luminous LBVs (such as R 71) correlate well with locations of red supergiants. They further argue that none of their confirmed LBVs are runaway stars. In a counter argument, Smith (2016) points out that runaway velocities predicted for the mass gainer in the binary evolution scenario are not necessarily high, in many cases ranging from slow (walkaway) velocities of a few $\mathrm{km} \mathrm{s}^{-1}$ to a few tens of $\mathrm{km} \mathrm{s}^{-1}$ (de Mink et al. 2014). The rejuvenated star also has a lifetime longer than would be expected for one of its luminosity if it were a single star, aiding and abetting the appearance of unusual isolation. Interestingly they attribute an LOS peculiar velocity of $-71 \mathrm{~km} \mathrm{~s}^{-1}$ to $\mathrm{R} 71$ arguing that this is consistent with it being a potential fast runaway (but we refer the reader to our discussion below, and the argument in Davidson et al. 2016 concerning this star's LOS velocity). While we cannot shed light on the LBV population of the LMC as a whole, we can address the nature of R 71. As discussed by Smith \& Tombleson (2015) it is the most isolated of the confirmed LBVs in the LMC. They estimate that there is no O-star within $300 \mathrm{pc}$, the closest being almost half a degree distant (or $450 \mathrm{pc}$ ) in projection.

Concerning R71's LOS velocity, we adopt a value of $204 \mathrm{~km} \mathrm{~s}^{-1}$ (see Table 1), compared to the value of $192 \mathrm{~km} \mathrm{~s}^{-1}$ used by Mehner et al. (2013) that is commonly also used by other authors. As they report, however, this latter estimate is based on the [Fe II] lines. However, Wobig (1993) reports a discrepancy between these lines and the absorption lines of neutral helium, metals, and higher series Balmer lines that implies a systemic radial velocity of $204 \pm 5 \mathrm{~km} \mathrm{~s}^{-1}$, a result also obtained by Wolf et al. (1981). Since the [Fe II] lines form in the expanding wind (as also noted by both Mehner et al. 2013; and Wolf et al. 1981), we adopt the higher radial velocity of $204 \mathrm{~km} \mathrm{~s}^{-1}$ that is weighted more towards the weaker photospheric absorption features in its spectrum that should be less influenced by its wind. We caution, however, that even these lines may well be slightly impacted and the actual systemic LOS velocity may be a little higher. Comparing with the predicted LOS velocity at this position of $238 \mathrm{~km} \mathrm{~s}^{-1}$ gives a peculiar LOS velocity of only $-34 \mathrm{~km} \mathrm{~s}^{-1}$. A comparison with the average red and yellow supergiant velocities from Neugent et al. (2012) produces a similar result, implying a mean velocity at this position of $\sim 235 \mathrm{~km} \mathrm{~s}^{-1}$ and a very slightly lower LOS peculiar velocity of $-31 \mathrm{~km} \mathrm{~s}^{-1}$.

Our results for R 71 indicate that both its proper motion and LOS velocity are most likely inconsistent with it being a fast runaway star (with peculiar velocity in the range $50-100 \mathrm{~km} \mathrm{~s}^{-1}$ ), having a transverse velocity of around $38 \mathrm{~km} \mathrm{~s}^{-1}$ (see Table 1) though with a formal uncertainty of $\pm 34 \mathrm{~km} \mathrm{~s}^{-1}$ (but we note that more realistic errors may be roughly half this value). At this velocity it would take almost $12 \mathrm{Myr}$ to travel the $450 \mathrm{pc}$ from the nearest grouping of OB stars, or about $6 \mathrm{Myr}$ assuming the uncertainty adds to the velocity (or $9 \mathrm{Myr}$ with our more optimistic error estimate). These numbers are difficult to reconcile with single-star main-sequence lifetimes of approximately 8,5 , 4, and 3 Myr for stars with initial masses of 20, 30, 40, and $60 M_{\odot}$ (Brott et al. 2011).

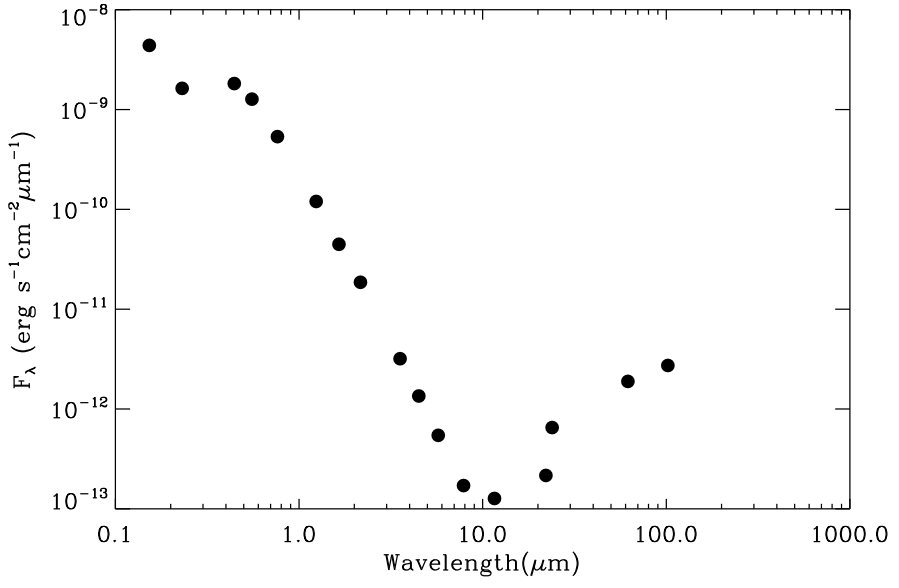

Fig. 5. Spectral energy distribution of the candidate hypervelocity star Sk-67 2 showing its infrared excess from about 10 to $100 \mu \mathrm{m}$ that is a signature of dust emission and a possible bow-shock (based on Spitzer, WISE, and IRAS data).

\section{2. $S k-672(=H D E 270754=H I P 22237)$}

Due to its moderately high extinction of $E(B-V) \sim 0.2$, Sk -67 2 has been the subject of many observations with various facilities as a means of studying the interstellar medium of the LMC (see Welty et al. 2006, for an example). The star is also classified by Fitzpatrick (1991) as belonging to those B-type supergiants, denoted as $N$ weak or $B C$, that appear nitrogen deficient with respect to the morphologically normal nitrogen rich supergiants. We find that this isolated star (it lies at the north-west edge of the galaxy, see Fig. 2) has a very large peculiar velocity of $359 \mathrm{~km} \mathrm{~s}^{-1}$ (Table 1) in a direction away from the LMC.

This star was omitted from the vdMS sample, in part because its peculiar proper motion implies that it cannot help constrain the overall LMC rotation field. However, they also noted that this star has the second highest astrometric_excess_noise parameter $a$ in the sample, after Sk-71 42 (see Sect. 3.3). However, while the latter star $(a=2.58)$ is truly a strong outlier, this is not the case for Sk-67 $2(a=1.11)$. The remaining 29 sample stars have a mean $\langle a\rangle=0.51$ with an RMS of 0.18 . There are two other stars in the sample with $a \sim 0.9$, HIP 22849 and 27868, and these do not have unusual proper motions (see Table 1). Therefore, the astrometric_excess_noise for Sk-67 2 does not necessarily imply that its measured TGAS proper motion must suffer from unidentified systematics. It is consistent with being an $\sim 3 \sigma$ random Gaussian outlier in the overall distribution. We also note that Sk-67 2 has a strong mid- and far-infrared excess (Bonanos et al. 2009; see also Fig. 5) that would also be consistent with a high peculiar velocity if that motion were to drive a bow shock as the star ploughs through the interstellar medium.

We have a high resolution FEROs echelle spectrum of this object obtained in 2004, and we have extracted high resolution UVES echelle spectra from the European Southern Observatory (ESO) archive obtained in 2001 and 2012. Radial velocities measured from the weak metal lines in these data lead to a radial velocity of $320 \pm 1 \mathrm{~km} \mathrm{~s}^{-1}$ for all three epochs. Comparing our measured radial velocity with the predicted mean LOS velocity from van der Marel \& Kallivayalil (2014) at this position of $277 \mathrm{~km} \mathrm{~s}^{-1}$ leads to a peculiar velocity of $+43 \mathrm{~km} \mathrm{~s}^{-1}$ (comparison with the average red and yellow supergiant velocities from Neugent et al. 2012 leads to a similar peculiar LOS velocity of $+49 \mathrm{~km} \mathrm{~s}^{-1}$ ). We also note that van der Marel \& Kallivayalil (2014) also cite a velocity dispersion for the young massive stars 
in the LMC of $11.6 \mathrm{~km} \mathrm{~s}^{-1}$ implying that Sk-67 2 is a clear $\sim 4 \sigma$ outlier, though perhaps not such an obvious outlier in LOS as in proper motion. Using instead $\sigma=20 \mathrm{~km} \mathrm{~s}^{-1}$ from Fig. 3 implies it is a $2.3 \sigma$ outlier.

Based on the above evidence we argue that this star is a true LMC member and not some peculiar foreground Halo object and, since its velocity vector is almost parallel to the disk plane (see below), it is highly unlikely that it is a more distant star of unexplained origin or nature. To understand whether or not Sk-67 2 is bound we must compare its velocity with the escape velocity of the LMC. While this is currently uncertain, we can obtain an approximate value by comparison with a Milky Way value of $533 \mathrm{~km} \mathrm{~s}^{-1}$ as derived from the Radial Velocity Experiment (RAVE) survey (Piffl et al. 2014). The ratio of these galaxies' total dark halo masses of 0.061 (Guo et al. 2010) then implies an escape velocity for the LMC of $\sim 131 \mathrm{~km} \mathrm{~s}^{-1}$. Alternatively if we directly compare circular velocities for the LMC (92 $\mathrm{km} \mathrm{s}^{-1}$, van der Marel \& Kallivayalil 2014) and Milky Way (239 $\mathrm{km} \mathrm{s}^{-1}$, McMillan 2011) we obtain an escape velocity of around $205 \mathrm{~km} \mathrm{~s}^{-1}$. Therefore Sk-67 2 is clearly unbound with respect to the LMC and, as we argue below, a possible hypervelocity star.

While Sk-67 2 is unbound its velocity is still significantly below the surface escape velocity of a main sequence star of its approximate mass and therefore it is an ambiguous candidate for a hypervelocity star since there are theoretical scenarios in which fast runaway stars, or hyper-runaway stars, might be created by classical runaway star scenarios. However, $N$-body simulations of dynamical ejection from massive clusters (Perets \& Šubr 2012) predict very low rates of production of hyper-runaway stars, while binary evolution models have difficulty producing any runaway stars with velocities in excess of $\sim 200 \mathrm{~km} \mathrm{~s}^{-1}$ (Eldridge et al. 2011). Based on current models therefore it would appear that the hyper-runaway scenario is not a promising explanation of the nature of Sk-67 2 .

One can calculate the three-dimensional position and threedimensional velocity vectors of Sk-672 in an LMC-centric frame using the approach used in van der Marel et al. (2002, this is a one-parameter family depending on the star's actual distance). The minimum angle between the star's position and velocity vectors in this frame is $5.3 \pm 5.2$ degrees, attained for a distance that is $50.6 \mathrm{kpc}$ (i.e. $0.5 \mathrm{kpc}$ larger than the distance of the LMC, but $0.5 \mathrm{kpc}$ in front of the inclined LMC disc at this location). The uncertainty in this angle is dominated by the uncertainty in the position of the LMC centre $(\sim 0.3$ deg per coordinate), which was taken from the joint fit to the HST and TGAS PM data in vdMS. In other words, its velocity vector is almost aligned with the LMC centre, as would be expected for a hypervelocity star ejected by a central massive black hole. We note here, however, that the star is $\sim 3.5 \mathrm{kpc}$ from the centre of the LMC implying that if the star distance is such that it is $\pm 3.5 \mathrm{kpc}$ from the LMC disc plane, then the angle between the velocity vector and the vector to the LMC centre would be $\sim 45$ degrees.

While no such black hole is known to exist, the presence of a black hole of mass $\leq 10^{7} M_{\odot}$ cannot be ruled out by the observed velocity field (Boyce et al. 2017). Interestingly Boubert \& Evans (2016) have suggested that the presence of a central massive black hole in the LMC might naturally explain the clustering of known hypervelocity stars in the constellations Leo and Sextens, provided that there is an as yet undiscovered southern population loosely focused on the LMC.

Given that its lifetime (below) clearly rules out a Milky Way origin, it is tempting to attribute membership of this population of hypervelocity stars to Sk-67 2. However, there are still some issues with this explanation. For example, its distance from the centre of the LMC is $\sim 3.5 \mathrm{kpc}$ that would take $\sim 10 \mathrm{Myr}$ to traverse at its current velocity. Even allowing for the fact that we have ignored the effect of the LMC gravitational potential in estimating its flight time, implying this is an upper limit, it is still more than a factor of two longer than its likely main sequence lifetime. If, like R 71, this star is a product of binary evolution, having been a hypervelocity binary ejected from a massive black hole at the centre of the LMC, then indeed there might be evolutionary channels that could be consistent with its current position. On the other hand, this discrepancy between lifetime and flight-time may provide some support for the hypothesis that some hypervelocity stars originate via interaction with an intermediate mass black hole (IMBH) as proposed by Przybilla et al. (2008) for the star HE0437-5439, thought to have originated from the LMC. However, the LMC is not known to host a confirmed IMBH either.

Stars in the LMC with unusual line-of-sight kinematics were previously reported by Olsen et al. (2011). These stars have velocity residuals up to $\sim 100 \mathrm{~km} \mathrm{~s}^{-1}$, and lower metallicities than typical for the LMC. They were interpreted as a population of accreted Small Magellanic Cloud (SMC) stars. The threedimensional velocity difference between the LMC and SMC is $128 \pm 32 \mathrm{~km} \mathrm{~s}^{-1}$ (Kallivayalil et al. 2013), consistent with the scale of the observed residual velocities for these stars. By contrast, Sk-67 2 has a velocity residual of $\sim 360 \mathrm{~km} \mathrm{~s}^{-1}$ compared to the LMC. Moreover, direct comparison to the known threedimensional velocity of the SMC implies a residual velocity of $\sim 336 \mathrm{~km} \mathrm{~s}^{-1}$ with respect to that galaxy. Therefore, Sk-67 2 cannot have an SMC origin (which would also be hard to reconcile with its short main sequence lifetime).

Finally in this subsection we note that the TGAS proper motion is essentially derived from the difference between the HIPPARCos and Gaia positions of this object (since its parallax is negligible). One might suppose that some perturbation of the point-spread-function, such as the presence of a close visual companion, might cause an error in the estimated position. However, Sk-67 2 is in a sparsely populated part of the LMC and our high resolution FEROS spectrum shows no evidence for a bright companion. Further, to cause a spurious proper motion of $\sim 1 \mathrm{mas} / \mathrm{yr}$ in TGAS, one would require an offset of $\sim 20$ mas in the Gaia astrometry. This is of the order of one-fifth of an AL/AC-average pixel and in addition this offset would have to be consistent for the different scan angles, which seems rather unlikely. In this context we also note that very good agreement is found between proper motions derived from the HST and TGAS for the Magellanic Clouds (vdMS) and Globular Clusters in the Milky Way (Watkins \& van der Marel 2017). We conclude that Sk-67 2 is a candidate hypervelocity star that if confirmed, by for example Gaia Data Release 2 (DR2), would warrant further investigation as to the nature of its origin.

\section{3. $S k-7142(=H D E 269660=H I P 25815)$}

The TGAS proper motions imply a peculiar velocity of $\sim 150 \mathrm{~km} \mathrm{~s}^{-1}$ for Sk-71 42, but in contrast to Sk-672 it is in a very crowded region of the LMC and lies (in projection) within the environment of the nebula and supernova remnant LHA 120-N206 that hosts many massive O-type stars. Also, by contrast, this star has astrometric_excess_noise = 2.58 , substantially above the values of the rest of the present sample. Also, comparing our measured radial velocity of $238 \mathrm{~km} \mathrm{~s}^{-1}$ with the mean LOS velocity predicted by the model of van der Marel \& Kallivayalil (2014) at this position 
of $239 \mathrm{~km} \mathrm{~s}^{-1}$ gives a peculiar LOS velocity of only $-1 \mathrm{~km} \mathrm{~s}^{-1}$, while from the monitoring of Morrell et al. (1999) we know that this star has constant radial velocity. Comparison to the average red and yellow supergiant velocities of Neugent et al. (2012) yields a peculiar velocity of $-12 \mathrm{~km} \mathrm{~s}^{-1}$. The lack of a peculiar LOS velocity, together with its unusually large astrometric_excess_noise parameter suggests that we must await further data on this object from Gaia DR2 before speculating further on its nature.

\section{Summary}

We have shown that the Gaia TGAS catalogue is even now, with DR1, able to provide important dynamical constraints on a subset of the visually brightest massive stars in the LMC which can be used to address their nature as potential walkaway, runaway, or hypervelocity stars. Specific conclusions concerning this sample include;

- Most of these very luminous stars are not runaways, the outliers mostly have peculiar velocities of less than $50 \mathrm{~km} \mathrm{~s}^{-1}$.

- R71 is rather unique in its isolation and we have shown that its peculiar space velocity is only moderately discrepant from its environment, which is difficult to reconcile with a single-star evolutionary scenario. A potential solution to this dilemma might be that this particular LBV is indeed the result of binary evolution but is the evolved product of a slow runaway binary (either a rejuvenated mass gainer or a stellar merger).

- The isolated B1.5 $\mathrm{Ia}^{+}$supergiant Sk-672 is found to be a candidate hypervelocity star with a peculiar velocity of $\sim 360 \mathrm{~km} \mathrm{~s}^{-1}$ directed radially away from the LMC centre, suggesting possible ejection by an as yet undiscovered central black hole. However, its main sequence lifetime is difficult to reconcile with the likely flight-time suggesting alternative hypotheses for the origin of its velocity will need to be explored.

Acknowledgements. This work has made use of data from the ESA space mission Gaia (http: //www . cosmos . esa.int/gaia), processed by the Gaia Data Processing and Analysis Consortium (DPAC, http://www . cosmos.esa.int/ web/gaia/dpac/consortium). Funding for the DPAC has been provided by national institutions, in particular the institutions participating in the Gaia Multilateral Agreement. D.J.L. thanks Hassan Siddiqui, Uwe Lammers, and Jose Hernandez of the ESAC Gaia Science Operations Centre for useful discussions. This research made use of Simbad and Vizier provided by CDS, Strasbourg; ESASky, developed by the ESAC Science Data Centre (ESDC); the ESO archive at Garching; and TOPCAT. J. Sahlmann was supported by an ESA Research Fellowship in Space Science.

\section{References}

Arp, H. 1992, MNRAS, 258, 800

Bestenlehner, J. M., Vink, J. S., Gräfener, G., et al. 2011, A\&A, 530, L14
Blaauw, A. 1961, Bull. Astron. Inst. Netherlands, 15, 265

Bonanos, A. Z., Massa, D. L., Sewilo, M., et al. 2009, AJ, 138, 1003

Boubert, D., \& Evans, N. W. 2016, ApJ, 825, L6

Boyce, H., Lützgendorf, N., van der Marel, R. P., et al. 2017, ApJ, submitted [arXiv: 1612.00045]

Brott, I., de Mink, S. E., Cantiello, M., et al. 2011, A\&A, 530, A115

Brown, W. R. 2015, ARA\&A, 53, 15

Davidson, K., Humphreys, R. M., \& Weis, K. 2016, ArXiv e-prints [arXiv: 1608.02007]

de Mink, S. E., Brott, I., Cantiello, M., et al. 2012, in Proc. Scientific Meeting in Honor of Anthony F. J. Moffat, eds. L. Drissen, C. Robert, N. St-Louis, \& A. F. J. Moffat, ASP Conf. Ser., 465, 65

de Mink, S. E., Sana, H., Langer, N., Izzard, R. G., \& Schneider, F. R. N. 2014, ApJ, 782, 7

Eldridge, J. J., Langer, N., \& Tout, C. A. 2011, MNRAS, 414, 3501

Evans, C. J., Walborn, N. R., Crowther, P. A., et al. 2010, ApJ, 715, L74

Fitzpatrick, E. L. 1991, PASP, 103, 1123

Freedman, W. L., Madore, B. F., Gibson, B. K., et al. 2001, ApJ, 553, 47

Gaia Collaboration (Brown et al.) 2016a, A\&A, 595, A2

Gaia Collaboration (Prusti et al.) 2016b, A\&A, 595, A1

Guo, Q., White, S., Li, C., \& Boylan-Kolchin, M. 2010, MNRAS, 404, 111

Gvaramadze, V. V., Kroupa, P., \& Pflamm-Altenburg, J. 2010, A\&A, 519, A33

Hénault-Brunet, V., Evans, C. J., Sana, H., et al. 2012, A\&A, 546, A73

Humphreys, R. M., Weis, K., Davidson, K., \& Gordon, M. S. 2016, ApJ, 825, 64

Kallivayalil, N., van der Marel, R. P., Besla, G., Anderson, J., \& Alcock, C. 2013, ApJ, 764, 161

Köhler, K., Langer, N., de Koter, A., et al. 2015, A\&A, 573, A71

Kroupa, P., \& Bastian, U. 1997, New Astron., 2, 77

Lindegren, L., Lammers, U., Bastian, U., et al. 2016, A\&A, 595, A4

McMillan, P. J. 2011, MNRAS, 414, 2446

Mehner, A., Baade, D., Rivinius, T., et al. 2013, A\&A, 555, A116

Morrell, N. I., Niemela, V. S., Barbá, R. H., et al. 1999, in Rev. Mex. Astron. Astrofis. Conf. Ser. 8, eds. N. I. Morrell, V. S. Niemela, \& R. H. Barbá, 149

Neugent, K. F., Massey, P., Skiff, B., \& Meynet, G. 2012, ApJ, 749, 177

Olsen, K. A. G., Zaritsky, D., Blum, R. D., Boyer, M. L., \& Gordon, K. D. 2011, ApJ, 737, 29

Parker, R. J., \& Goodwin, S. P. 2007, MNRAS, 380, 1271

Perets, H. B., \& Šubr, L. 2012, ApJ, 751, 133

Piffl, T., Scannapieco, C., Binney, J., et al. 2014, A\&A, 562, A91

Platais, I., van der Marel, R. P., Lennon, D. J., et al. 2015, AJ, 150, 89

Poveda, A., Ruiz, J., \& Allen, C. 1967, Boletin de los Observatorios Tonantzintla y Tacubaya, 4,86

Prinja, R. K., \& Schild, H. 1991, A\&A, 249, 428

Przybilla, N., Nieva, M. F., Heber, U., et al. 2008, A\&A, 480, L37

Sanduleak, N. 1970, Contributions from the Cerro Tololo Inter-American Observatory, 89

Shore, S. N., \& Sanduleak, N. 1984, ApJS, 55, 1

Smith, N. 2016, MNRAS, 461, 3353

Smith, N., \& Tombleson, R. 2015, MNRAS, 447, 598

van der Marel, R. P., Alves, D. R., Hardy, E., \& Suntzeff, N. B. 2002, AJ, 124, 2639

van der Marel, R. P., \& Kallivayalil, N. 2014, ApJ, 781, 121

van der Marel, R. P., \& Sahlmann, J. 2016, ApJ, 832, L23

Watkins, L. L., \& van der Marel, R. P. 2017, ApJ, 839, 89

Welty, D. E., Federman, S. R., Gredel, R., Thorburn, J. A., \& Lambert, D. L. 2006, ApJS, 165, 138

Wobig, D. 1993, Master's Thesis, Sternwarte der Ludwig-MaximiliansUniversität München

Wolf, B., Appenzeller, I., \& Stahl, O. 1981, A\&A, 103, 94 